\title{
Conjugated heat transfer in circular microchannels with slip flow and axial diffusion effects
}

\author{
Diego C. Knupp ${ }^{a}, *$, Fabricio S. Mascouto ${ }^{a}$, Luiz A.S. Abreu ${ }^{a}$, Carolina P. Naveira-Cotta ${ }^{b}$, \\ Renato M. Cotta ${ }^{\mathrm{b}}$

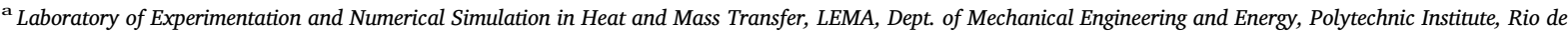 \\ Janeiro State University, IPRJ/UERJ, Rua Bonfim 25, Vila Amelia, Nova Friburgo, RJ 2625-570, Brazil \\ ${ }^{\mathrm{b}}$ LabMEMS - Laboratory of Nano \& Microfluidics and Microsystems, Mechanical Engineering Department - PEM, POLI/COPPE, Nanoengineering Department - PENT, \\ COPPE, Federal University of Rio de Janeiro, UFRJ, Cx. Postal 68503 - Cidade Universitária, 21945-970 Rio de Janeiro, RJ, Brazil
}

\section{A R T I C L E I N F O}

\section{Keywords:}

Conjugated problem

Slip flow

Temperature jump

Generalized Integral Transform Technique

Convergence enhancement

Single domain formulation

\begin{abstract}
A B S T R A C T
An integral transforms analysis is undertaken for conjugated heat transfer in circular microchannels with laminar gaseous flow in the slip flow regime. The solution methodology is based on the Generalized Integral Transform Technique applied to a single domain formulation that models the coupled heat transfer phenomena at the fluid stream and at the channel wall. The single domain formulation results in just one partial differential equation for the energy balance, making use of spatially variable coefficients with abrupt transitions, and accounting for the temperature jump at the interface due to the Knudsen numbers within the slip flow regime. This work extends the single domain formulation strategy, not a priori applicable to problems with discontinuities, by considering a very thin fictitious layer at the fluid-wall interface region, so as to mathematically represent an equivalence to the temperature jump. An integral balance technique for enhancing the convergence of the eigenfunctions is employed, so as to achieve more accurate results and improve convergence for the so derived multiscale problem. The results obtained are critically compared against a dedicated finite difference numerical solution for the original multi-region problem. Results for the Nusselt number are presented in order to investigate its behavior with respect to different Péclet and Knudsen numbers, and different wall thicknesses values, confirming the importance of the combined effects of slip flow, axial conduction and heat transfer conjugation in the analysis.
\end{abstract}

\section{Introduction}

Several earlier works in the analysis of thermal microsystems led to the observation of discrepancies between the experimental results and classical correlations or simulations for the associated heat transfer coefficients, as reviewed in [1]. These discrepancies are mainly related to the adoption of classical hypothesis employed in modeling macroscale problems, which may no longer be valid when dealing with heat and fluid flow in microsystems [2]. In such cases, due to the very small characteristic lengths involved, it is required to modify and extend the flow and convective heat transfer modeling, in comparison to the usual simplified macro-scale formulations [3].

A few analytically based solutions have been provided in the literature aiming at the analysis of micro-scale convective heat transfer, such as for instance for heat transfer with slip flow in circular microtubes [4,5], rectangular and parallel plates microchannels [6-8], and the investigation of viscous heating and fluid property variation [9]. Nevertheless, the effects of axial diffusion due to the low Péclet numbers involved and the conduction-convection conjugation effect due to the microsystem substrate, have been avoided in such analytical developments. In ref. [10] the conjugation effect was taken into consideration for heat transfer in a parallel plates microchannel, employing the Generalized Integral Transform Technique (GITT) [11,12] in combination with a single domain formulation. This reformulation strategy was proposed towards rewriting multi-region problems, such as those in conjugated conduction-convection heat transfer, into single region problems with space variable thermophysical properties and source terms, allowing for a single integral transformation operation over the

\footnotetext{
* Corresponding author.

E-mail address: diegoknupp@iprj.uerj.br (D.C. Knupp).
} 


$\begin{array}{ll}\text { Nomenclature } \\ c_{p, f} & \text { fluid specific heat at constant pressure } \\ c_{v, f} & \text { fluid specific heat at constant volume } \\ h_{z} & \text { local heat transfer coefficient } \\ k & \text { thermal conductivity } \\ K & \text { dimensionless thermal conductivity } \\ \mathrm{Kn} & \text { Knudsen number } \\ K_{f i c} & \text { dimensionless thermal conductivity of the fictitious layer } \\ L_{z} & \text { microchannel length } \\ L_{f i c} & \text { dimensionless thickness of the fictitious layer } \\ M & \text { truncation order of the eigenfunction expansion (eigen- } \\ & \text { value problem solution) } \\ N & \text { truncation order of the temperature eigenfunction expan- } \\ & \text { sion } \\ \mathrm{Nu} & \text { Nusselt number } \\ N_{\psi} & \text { norm corresponding to temperature eigenfunction, } \psi \\ N_{\Omega} & \text { norm corresponding to auxiliary eigenfunction, } \Omega \\ \mathrm{Pe} & \text { Péclet number } \\ \mathrm{Pr} & \text { Prandtl number } \\ q_{w} & \text { wall heat flux } \\ r & \text { radial position } \\ R & \text { dimensionless radial position } \\ \mathrm{Re} & \text { Reynolds number } \\ T & \text { temperature } \\ T_{\mathrm{w}} & \text { prescribed temperature at the external wall } \\ T_{\mathrm{in}} & \text { prescribed temperature at the inlet } \\ u & \text { velocity field } \\ z & \text { longitudinal position } \\ Z & \text { dimensionless longitudinal position } \\ & \end{array}$

\section{Greek letters}

$\begin{array}{ll}\alpha_{m} & \text { tangential momentum accommodation coefficient } \\ \alpha_{t} & \text { thermal accommodation coefficient } \\ \beta_{t} & \text { wall temperature jump coefficient } \\ \beta_{v} & \text { wall velocity slip coefficient } \\ \gamma & \text { specific heat ratio } \\ \lambda & \text { molecular mean free path } \\ \eta & \text { eigenvalues corresponding to auxiliary eigenfunction, } \Omega \\ \Omega & \text { auxiliary eigenfunctions } \\ \mu & \text { eigenvalues corresponding to temperature eigenfunction, } \\ & \psi \\ \rho & \text { temperature eigenfunctions } \\ \rho & \text { specific mass } \\ & \text { dimensionless temperature }\end{array}$

Subscripts and superscripts

$\begin{array}{ll}a v & \text { average } \\ f & \text { quantities related to the fluid region } \\ \text { fic } & \text { quantities related to the fictitious layer } \\ o & \text { quantities at the outer wall } \\ s & \text { quantities related to the solid region } \\ i & \text { quantities at the inner wall } \\ j, l, m, n & \begin{array}{l}\text { order of eigenquantitites } \\ \text { domain including the fictitious layer } \\ *\end{array} \\ \sim & \begin{array}{l}\text { normalized eigenfunction } \\ \text { integral transform }\end{array}\end{array}$

whole physical domain. This hybrid approach has also been demonstrated quite successfully in dealing with axial diffusion effects [13], complex configurations and irregular regions [14-16], as reviewed in [3], automatically satisfying heat flux and temperature continuity conditions at the interfaces, and without the need for domain decomposition schemes. Nonetheless, rarefaction effects were not included in those conjugated heat transfer studies [10,13-16].

Hence, the present work addresses an analytically based solution extending the analysis performed in [10,13-16] in order to simultaneously handle all these typical micro-scale effects: (i) the slip boundary condition in opposition to the classical no-slip boundary condition, together with the interfacial temperature discontinuity (temperature jump condition); (ii) the inclusion of the axial conduction term in the fluid energy equation; and (iii) the consideration of the conduction-convection conjugation effect. Despite the importance of modeling and analytically handling these three extensions of the classical Graetz problem at the microscale, the analysis of their combined effects has been somehow overlooked in the earlier literature, with a few relevant exceptions that employed discrete numerical methods and included simultaneously developing flow conditions $[17,18]$.

Actually, in ref. [19] the combination of the single domain formulation and the integral transforms approach was first extended to solve the conjugated heat transfer problem within the slip flow regime, when a fictitious very thin layer between the fluid region and the actual channel wall was introduced, in order to impose the desired thermal resistance between the fluid and the wall, and thus model the temperature jump at the interface. This fictitious solid layer needs to be kept much thinner than the characteristic length of the channel, so as to minimize the perturbation to the original problem geometry. However, such a solution based on the eigenfunction expansion of the temperature field, obtained through the integral transformation of the heat transfer problem, ends up by involving the solution of an eigenvalue problem with abrupt and multiscale variations on the governing space variable coefficients in the single domain formulation, resulting in undesirable slower convergence rates.

Therefore, based on a very recent development regarding the convergence acceleration of eigenfunction expansions for Sturm-Liouville problems $[20,21]$, employing an integral balance analytical procedure $[11,12]$, the present work proposes the combination of the single domain formulation and the integral transforms method to accurately and efficiently handle the conjugated heat transfer problem within circular microchannels in the slip flow regime, including axial diffusion effects. For verification purposes, a steady or quasi-steady state test problem with hydrodynamically fully developed and thermally developing flow is addressed. A dedicated finite difference simulation is also implemented for the original multi-region problem [22], allowing for critical comparisons. In order to investigate the influence of the microscale effects on convective heat transfer, the Nusselt number is computed for different combinations of wall thicknesses, Péclet and Knudsen numbers.

\section{Problem formulation and solution methodology}

Consider the incompressible gas flow within a circular microchannel with length $L_{z}$, cross-section with inner radius $r_{i}$ and outer radius $r_{o}$, as illustrated in Fig. 1, undergoing convective heat transfer due to a prescribed temperature $T_{w}$ at the external wall, different from the inlet temperature $T_{i n}$. The channel wall is considered to participate 


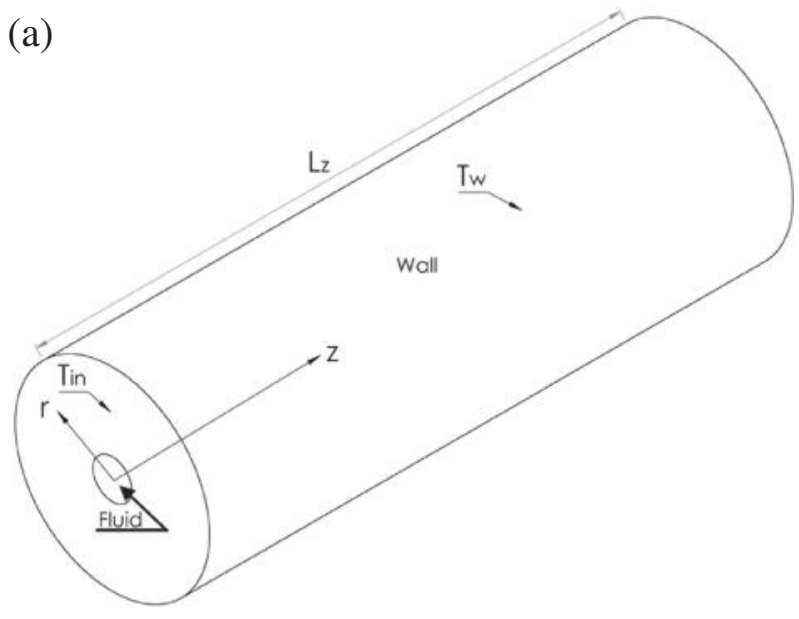

(b)

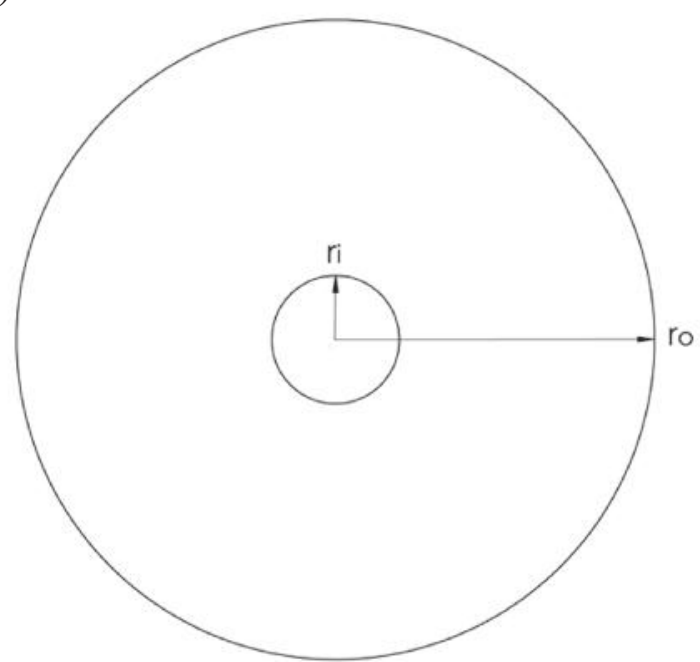

Fig. 1. (a) Schematic representation of the conjugated problem. (b) Microchannel crosssection.

on the heat transfer process through both radial and axial heat conduction. Besides, the Knudsen and Péclet numbers are such that the problem falls within the region of validity of the first order slip flow model and the axial diffusion effects in the fluid cannot be neglected.

The flow is here considered to be hydrodynamically fully developed, in which the gas enters the microchannel with a fully developed velocity profile $u_{f}(r)$, while a thermally developing region is established. The dimensionless formulation of the multi-region heat transfer problem, given by the coupled convection and conduction equations, is written as:

Heat convection equation (fluid region):

$$
\begin{aligned}
U_{f}(R) \frac{\partial \theta_{f}(R, Z)}{\partial Z}=\frac{K_{f}}{\mathrm{Pe}^{2}} \frac{\partial^{2} \theta_{f}(R, Z)}{\partial Z^{2}}+4 R_{i}^{2} \frac{1}{R} \frac{\partial}{\partial R}\left(R \frac{\partial \theta_{f}(R, Z)}{\partial R}\right), \\
0<R<R_{i}, \quad 0<Z<L_{Z}
\end{aligned}
$$

$\theta_{f}(R, 0)=1 ;\left.\quad \frac{\partial \theta_{f}}{\partial Z}\right|_{Z=L Z}=0$ $\left.\frac{\partial \theta_{f}}{\partial R}\right|_{R=0}=0 ;\left.\quad 2 R_{i} \beta_{t} \operatorname{Kn} \frac{\partial \theta_{f}}{\partial R}\right|_{R=R_{i}}+\theta_{f}\left(R_{i}, Z\right)=\theta_{S}\left(R_{i}, Z\right)$

Heat conduction equation (microchannel wall):

$4 R_{i}^{2} \frac{1}{R} \frac{\partial}{\partial R}\left(R \frac{\partial \theta_{S}(R, Z)}{\partial R}\right)+\frac{1}{\mathrm{Pe}^{2}} \frac{\partial^{2} \theta_{S}(R, Z)}{\partial Z^{2}}$

$=0, \quad R_{i}<R<1, \quad 0<Z<L_{Z}$

$\theta_{S}(R, 0)=1 ;\left.\quad \frac{\partial \theta_{s}}{\partial Z}\right|_{Z=L_{Z}}=0$

$\left.K_{s} \frac{\partial \theta_{s}}{\partial R}\right|_{R=R_{i}}=\left.K_{f} \frac{\partial \theta_{f}}{\partial R}\right|_{R=R_{i}} ; \quad \theta_{s}(1, Z)=0$

The temperature jump condition at the interface is modeled by Eq. $(1 \mathrm{~d}, \mathrm{e})$ and the heat flux continuity is modeled by Eq. $(2 \mathrm{~d}, \mathrm{e})$. The following dimensionless groups have been employed in Eqs. (1a)-(1d,e) and Eqs. (2a)-(2d,e):

$$
\begin{aligned}
& Z=\frac{z /\left(2 r_{i}\right)}{\operatorname{Re} \operatorname{Pr}} ; \quad R=\frac{r}{r_{o}} ; \quad U_{f}=\frac{u_{f}}{u_{a v}} ; \quad \theta=\frac{T-T_{w}}{T_{i n}-T_{w}} ; \quad K=\frac{k}{k_{f}} ; \quad \operatorname{Re} \\
& =\frac{u_{a v}\left(2 r_{i}\right)}{v} \\
& \operatorname{Pr}=\frac{\nu \rho_{f} c_{p, f}}{k_{f}} ; \quad \operatorname{Pe}=\operatorname{RePr}=\frac{u_{a v}\left(2 r_{i}\right) \rho_{f} c_{p, f}}{k_{f}} ; \quad \beta_{t}=\frac{\left(2-\alpha_{t}\right)}{\alpha_{t}} \frac{2 \gamma}{(\gamma+1)} \frac{1}{\operatorname{Pr}} ; \\
& \mathrm{Kn}=\frac{\lambda}{2 r_{i}}
\end{aligned}
$$

where the subscript $f$ denotes quantities related to the fluid, $s$ denotes quantities related to the solid (microchannel wall), $\alpha_{t}$ is the thermal accommodation coefficient, $\gamma=c_{p, f} / c_{v, f}$ is the specific heat ratio, and $\lambda$ is the molecular mean free path. The dimensionless velocity profile is given by [5]:

$U_{f}(R)=\frac{3\left[1-\left(R / R_{i}\right)^{2}+4 \mathrm{Kn} \beta_{v}\right]}{2\left(1+6 \mathrm{Kn} \beta_{v}\right)}$ with $\beta_{v}=\frac{2-\alpha_{m}}{\alpha_{m}}$

where $\beta_{v}$ is the wall velocity slip coefficient and $\alpha_{m}$ is the tangential momentum accommodation coefficient.

In order to write the conjugated problem given by Eqs. (1a)-(1d,e) and Eqs. (2a)-(2d,e) in a single domain formulation, while still accounting for the temperature jump interface condition, it is considered a fictitious thin layer between the fluid stream and the channel wall, in such a way that this fictitious layer can be chosen with dimensionless thickness and thermal conductivity, $L_{f i c}$ and $K_{f i c}$, respectively, so as to impose the equivalent thermal resistance [19]. Considering that this fictitious layer undergoes steady state heat conduction in the radial direction only, and considering the boundary condition given by Eq. $(1 \mathrm{~d}, \mathrm{e})$, a thermal resistance analysis yields the following relation:

$K_{f i c}=\ln \left(\frac{R_{i}+L_{f i c}}{R_{i}}\right) \frac{1}{2 \beta_{t} \mathrm{Kn}}$

One should observe that even though any arbitrary value could be set for $L_{f i c}$ in Eq. (5) yielding the adequate thermal conductivity for the fictitious layer, according to the required thermal resistance, this thickness should be ideally chosen with $L_{f i c} \rightarrow 0$, to interfere as minimum as possible in the original geometry and on the overall heat transfer process.

The conjugated problem simulating the temperature jump interface condition can now be written as a single domain formulation with space variable coefficients. However, instead of directly solving the reformulated steady state problem presented in Eqs. (1a)-(1d,e) and (2a)-(2d,e), which upon integral transformation in the radial variable, 
would result in a boundary value problem for the transformed temperature along the longitudinal coordinate, it is computationally more convenient to employ the partial transformation scheme of the GITT approach, as detailed in [23], after introducing a pseudo transient parabolic formulation, with the convection term considered as a source term [13]. The single domain formulation is then written as:

$$
\begin{aligned}
\frac{\partial \theta^{*}}{\partial t}=4 R_{i}^{2} \frac{1}{R} \frac{\partial}{\partial R}\left(R K(R) \frac{\partial \theta^{*}}{\partial R}\right)+\frac{K_{a x}(R)}{\mathrm{Pe}^{2}} \frac{\partial^{2} \theta^{*}}{\partial Z^{2}}-U(R) \frac{\partial \theta^{*}}{\partial Z}, & \\
0 & <R<1+L_{f i c}, \quad 0<Z<L_{Z}, \quad t>0
\end{aligned}
$$

$\theta^{*}(R, 0, t)=1 ;\left.\quad \frac{\partial \theta^{*}}{\partial Z}\right|_{Z=L Z}=0$

$\left.\frac{\partial \theta^{*}}{\partial R}\right|_{R=0}=0 ; \quad \theta^{*}\left(1+L_{f i c}, Z, t\right)=0$

$\theta^{*}(R, Z, 0)=f(R, Z)$

where the initial condition $f(R, Z)$ can be any feasible function, preferably any approximation to the steady state solution, since the interest here is only in the steady state analysis, $t \rightarrow \infty$. Here, for the computational implementation, the initial temperature for the pseudo transient formulation was taken equal to the wall temperature, $f(R, Z)$ $=0$. The space variable coefficients in the single domain formulation in Eq. (6a), are defined as:

$$
\begin{aligned}
& U(R)=\left\{\begin{array}{l}
U_{f}(R), \text { if } 0 \leq R<R_{i} \\
0, \quad \text { if } R_{i} \leq R \leq 1+L_{f i c}
\end{array}, \quad K(R)=\left\{\begin{array}{l}
1, \quad \text { if } 0 \leq R<R_{i} \\
K_{f i c}, \text { if } R_{i} \leq R<R_{i}+L_{f i c} \\
k_{s} / k_{f}, \text { if } R_{i}+L_{f i c} \leq R \leq 1+L_{f i c}
\end{array},\right.\right. \\
& K_{a x}(R)= \begin{cases}1, & \text { if } 0 \leq R<R_{i} \\
0, & \text { if } R_{i} \leq R<R_{i}+L_{f i c} \\
k_{s} / k_{f}, & \text { if } R_{i}+L_{f i c} \leq R \leq 1+L_{f i c}\end{cases}
\end{aligned}
$$

It is worth noting that $K_{a x}(R)$ is introduced with zero thermal conductivity at the fictitious layer, so that this layer participates through heat conduction along the radial direction only, thus allowing for the thermal resistance analysis performed in deriving Eq. (5).

After the problem given by Eqs. (6a)-(6g-i) is solved, the desired temperature distribution can be readily obtained by considering the steady state solution, with $t$ sufficiently high, and removing the fictitious layer from the solution:

$\theta(R, Z)=\left\{\begin{array}{l}\theta^{*}(R, Z, t \rightarrow \infty), \text { if } 0 \leq R \leq R_{i} \\ \theta^{*}\left(R+L_{f i c}, Z, t \rightarrow \infty\right), \text { if } R>R_{i}\end{array}\right.$

So, whereas $\theta^{*}$ is defined for $0 \leq R \leq 1+L_{f i c}, \theta$ is defined for $0 \leq R \leq 1$, the original problem domain before introducing the fictitious layer. As demonstrated in a number of previous works $[13,15]$, the problem given by Eqs. (6a)-(6g-i) can be solved through the proposition of an eigenvalue problem containing the information regarding the different domains in its governing coefficients. For the problem addressed in this work, one may then choose the following eigenvalue problem:

$\frac{d}{d R}\left(R K(R) \frac{d \psi_{l}(R)}{d R}\right)+\mu_{l}^{2} R \psi_{l}(R)=0$

$\left.\frac{d \psi_{l}}{d R}\right|_{R=0}=0 ; \quad \psi_{l}\left(1+L_{f i c}\right)=0$

which allows for the definition of the following transform-inverse pair: transform: $\bar{\theta}_{l}^{*}(Z, t)=\int_{0}^{1+L_{f i c}} R \widetilde{\psi}_{l}(R) \theta^{*}(R, Z, t) d R$

inverse: $\theta^{*}(R, Z, t)=\sum_{l=1}^{\infty} \widetilde{\psi}_{l}(R) \bar{\theta}_{l}^{*}(Z, t)$

with the normalized eigenfunctions calculated as:

$\widetilde{\psi}_{l}(R)=\frac{\psi_{l}(R)}{N_{\psi, l}^{1 / 2}}, \quad$ with $N_{\psi, l}=\int_{0}^{1+L_{f i c}} R \psi_{l}^{2}(R) d R$

Operating on Eq. (6a) with $\int_{0}^{1+L_{\text {fic }}} R \widetilde{\psi}_{l}(R)(\cdot) d R$ and making use of the boundary conditions, we obtain the following one-dimensional transformed partial differential system:

$\frac{\partial \bar{\theta}_{l}^{*}(Z, t)}{\partial t}+\mu_{l}^{2} \bar{\theta}_{l}^{*}(Z, t)=\bar{g}_{l}\left(Z, t, \bar{\theta}^{*}\right), \quad l=1,2, \ldots$

with

$\bar{\theta}^{*}=\left[\bar{\theta}_{1}^{*}, \bar{\theta}_{2}^{*}, \ldots\right]$

where

$$
\begin{aligned}
\bar{g}_{l}\left(Z, t, \bar{\theta}^{*}\right)= & -\sum_{n=1}^{\infty} \frac{\partial \bar{\theta}_{n}}{\partial Z} \int_{0}^{1+L_{f i c}} R U(R) \widetilde{\psi}_{l} \widetilde{\psi}_{n} d R \\
& +\sum_{n=1}^{\infty} \frac{1}{\mathrm{Pe}^{2}} \frac{\partial^{2} \bar{\theta}_{n}}{\partial Z^{2}} \int_{0}^{1+L_{f i c}} R K_{a x}(R) \widetilde{\psi}_{l} \widetilde{\psi}_{n} d R
\end{aligned}
$$

with the following transformed boundary and initial conditions, obtained after operating on Eqs. (6b,c) and Eq. (6f) with $\int_{0}^{1+L_{f i c}} R \widetilde{\psi}_{l}(R)(\cdot) d R$ : $\bar{\theta}_{l}^{*}(0, t)=\int_{0}^{1+L_{\text {fic }}} R \widetilde{\psi}_{l}(R) d R ;\left.\quad \frac{\partial \bar{\theta}_{l}^{*}}{\partial Z}\right|_{Z=L Z}=0$

$\bar{\theta}_{l}^{*}(Z, 0)=\bar{f}_{l}(Z)=\int_{0}^{1+L_{f i c}} R \widetilde{\psi}_{l}(R) f(R, Z) d R$

System (11), after truncation to an order $N$, can be numerically solved to provide results for the transformed temperatures $\bar{\theta}_{l}^{*}(Z, t)$. The Mathematica software [24], for instance, provides the routine NDSolve which is able to solve this kind of partial differential system under automatic absolute and relative errors control.

Hence, the main task in this procedure is related to the solution of the eigenvalue problem given by Eqs. (8a)-(8b,c), which does not allow for a closed-form solution. Although the GITT itself can be readily employed to solve this eigenvalue problem $[3,13,15]$, the convergence rates can become fairly slow, due to the multiscale behavior of the governing coefficients, requiring the implementation of a convergence enhancement technique for computational savings, such as the Integral Balance procedure $[11,12]$, which can be employed to derive analytical expressions to accelerate the eigenfunctions expansion convergence $[20,21]$.

Starting with the analytical double integration of the original eigenvalue problem from the two boundaries to any arbitrary point in the domain, analytical expressions for the eigenfunction and its derivative are obtained, with dependence on the boundary values of both the eigenfunction and the associated derivative, and explicitly accounting for the space variable coefficients in the eigenfunction representation. Making use of the available boundary conditions in the original eigenvalue problem, the boundary values are eliminated from the derived 
expressions for the eigenfunction and its derivative. Following this procedure in solving the eigenvalue problem given by Eqs. (8a)-(8b,c), as described in details for a general Sturm-Liouville problem in [20], the following expressions with enhanced convergence are obtained for the eigenfunctions $\psi(R)$ and the derivatives $d \psi(R) / d R$ :

$\psi_{l}(R)=\mu_{l}^{2} \int_{R}^{1+L_{f i c}} \frac{1}{K\left(R^{\prime}\right)}\left[\int_{0}^{R^{\prime}} R^{\prime \prime} \psi_{l}\left(R^{\prime \prime}\right) d R^{\prime \prime}\right] d R^{\prime}$

$\frac{d \psi_{l}(R)}{d R}=-\frac{\mu_{l}^{2}}{R K(R)}\left[\int_{0}^{R} R^{\prime} \psi_{l}\left(R^{\prime}\right) d R^{\prime}\right]$

Then, consider an expansion representation for the original eigenfunctions, $\psi_{l}(R)$, appearing on the RHS of Eqs. (12a), (12b):

transform: $\bar{\psi}_{l, n}=\int_{0}^{1+L_{f i c}} R \widetilde{\Omega}_{n}(R) \psi_{l}(R) d R$

inverse: $\psi_{l}(R)=\sum_{n=1}^{\infty} \widetilde{\Omega}_{n}(R) \bar{\psi}_{l, n}$

which employs a simpler basis, such as provided by the following eigenvalue problem, with closed-form solution for the eigenfunctions $\Omega(R)$, and the corresponding eigenvalues $\eta$ :

$\frac{d}{d R}\left(R \frac{d \Omega_{n}(R)}{d R}\right)+\eta_{n}{ }^{2} R \Omega_{n}(R)=0$

$\left.\frac{d \Omega_{n}}{d R}\right|_{R=0}=0 ; \quad \Omega_{n}\left(1+L_{f i c}\right)=0$

The normalized auxiliary eigenfunctions are calculated as:

$\widetilde{\Omega}_{n}(R)=\frac{\Omega_{n}(R)}{N_{\Omega, n}^{1 / 2}}, \quad$ with $N_{\Omega, n}=\int_{0}^{1+L_{f i c}} R \Omega_{n}^{2}(R) d R$

Substituting the expansion representation given by Eq. (13b) into the RHS of Eqs. (12a), (12b) one obtains:

$\psi_{l}(R)=\mu_{l}^{2} \sum_{n=1}^{\infty} \bar{\psi}_{l, n} I B_{n}(R)$

$\frac{d \psi_{l}(R)}{d R}=-\frac{\mu_{l}^{2}}{K(R)} \sum_{n=1}^{\infty} \bar{\psi}_{l, n} I A_{n}(R)$

with

$I A_{n}(R)=\int_{0}^{R} R^{\prime} \widetilde{\Omega}_{n}\left(R^{\prime}\right) d R^{\prime}$,

$I B_{n}(R)=\int_{R}^{1+L_{f i c}} \frac{1}{K\left(R^{\prime}\right)} I A_{n}\left(R^{\prime}\right) d R^{\prime}$

$(16 \mathrm{c}, \mathrm{d})$

The integral transformation of Eq. (8a) can be achieved by operating on this equation with $\int_{0}^{1+L_{\text {fic }}}(\cdot) \widetilde{\Omega}_{m}(R) d R$. Then, substituting the integral balance expressions given by Eqs. (16a), (16b) for $\psi_{l}(R)$ and $d \psi_{l}(R) / d R$ appearing in this expression, yields:

$\left(\mathbf{A}-\mu^{2} \mathbf{B}\right) \bar{\Psi}=0$

with $A_{n, m}=\int_{0}^{1+L_{\text {fic }}} I A_{n}(R) \frac{d \widetilde{\Omega}_{m}(R)}{d R} d R$,

$B_{n, m}=-\int_{0}^{1+L_{f i c}} R I B_{n}(R) \widetilde{\Omega}_{m}(R) d R$

(18a-b)

where $I A_{n}(R)$ and $I B_{n}(R)$ are given by Eq. $(16 \mathrm{c}, \mathrm{d})$. This algebraic eigenvalue problem can be numerically solved to provide results for the eigenvalues $\mu_{l}$ and eigenvectors $\bar{\psi}_{l}$, which can be readily employed back in Eqs. (16a), (16b) to obtain analytical expressions for the eigenfunctions and related derivatives, $\psi_{l}(R)$ and $d \psi_{l}(R) / d R$, respectively.

Besides the GITT solution with integral balance for the single domain formulation just described, this work also reports some results obtained without the convergence acceleration technique, for comparison purposes. Moreover, a dedicated finite difference solution (secondorder accurate in $R$ and first-order accurate in $Z$ ) is implemented for the original multi-region problem given by Eqs. (1a)-(1d,e) and Eqs. (2a)-(2d,e), in order to provide a comparative analysis against the single domain integral transforms solution.

\subsection{Nusselt number}

The convective heat flux from the fluid to the wall is written as:

$q_{w}=h_{z}\left[T_{f, b}(z)-T_{s, i}(z)\right]$

where $h_{z}$ is the local heat transfer coefficient, $T_{f, b}(z)$ is the fluid bulk temperature and $T_{s, i}(z)$ is the inner wall temperature (interface with the fluid stream). The heat flux can also be written using Fourier's law:

$q_{w}=-\left.k_{f} \frac{\partial T_{f}(r, z)}{\partial r}\right|_{r=r_{i}}$

Equating Eq. (19) and Eq. (20) yields:

$h_{z}=-\left.k_{f} \frac{\partial T_{f}(r, z)}{\partial r}\right|_{r=r_{i}} \frac{1}{\left(T_{f, b}(z)-T_{s, i}(z)\right)}$

or in dimensionless form:

$h_{Z}=-\left.\frac{k_{f}}{r_{o}} \frac{\partial \theta_{f}(R, Z)}{\partial R}\right|_{R=R_{i}} \frac{1}{\left[\theta_{f, b}(Z)-\theta_{s}\left(R_{i}, Z\right)\right]}$

and the local Nusselt number is readily determined as:

$\mathrm{Nu}_{Z}=\frac{2 r_{i} h_{Z}}{k_{f}}=-\frac{\left.2 R_{i} \frac{\partial \theta_{f}(R, Z)}{\partial R}\right|_{R=R_{i}}}{\theta_{f, b}(Z)-\theta_{S}\left(R_{i}, Z\right)}$

The dimensionless fluid bulk temperature is given as:

$\theta_{f, b}(Z)=\frac{\int_{0}^{R_{i}} U_{f}(R) \theta_{f}(R, Z) R d R}{\int_{0}^{R_{i}} U_{f}(R) R d R}$

The derivative $\partial \theta_{f}(R, Z) /\left.\partial R\right|_{R_{i}}$ in Eq. (23a) is calculated combining the inverse formula, Eq. (9b), with the enhanced convergence expression for the eigenfunctions derivative, Eq. (12b), yielding:

Table 1

Convergence behavior of the GITT solution for the first five eigenvalues in problem (8) with $L_{f i c}=10^{-3}$ : (a) GITT with integral balance; (b) GITT without integral balance.

\begin{tabular}{llllll}
\hline$M$ & $\mu_{1}$ & $\mu_{2}$ & $\mu_{3}$ & $\mu_{4}$ & $\mu_{5}$ \\
\hline (a) & & & & & \\
10 & 2.544 & 4.305 & 6.910 & 9.776 & 11.665 \\
20 & 2.544 & 4.297 & 6.909 & 9.639 & 11.444 \\
30 & 2.544 & 4.296 & 6.909 & 9.627 & 11.436 \\
40 & 2.543 & 4.296 & 6.909 & 9.623 & 11.433 \\
50 & 2.543 & 4.295 & 6.909 & 9.620 & 11.431 \\
(b) & & & & & \\
500 & 2.566 & 4.589 & 6.923 & 10.015 & 11.737 \\
1000 & 2.565 & 4.571 & 6.922 & 9.994 & 11.714 \\
2000 & 2.557 & 4.455 & 6.916 & 9.841 & 11.577 \\
4000 & 2.549 & 4.361 & 6.912 & 9.709 & 11.483 \\
8000 & 2.546 & 4.324 & 6.910 & 9.656 & 11.450 \\
\hline
\end{tabular}


$\left.\frac{\partial \theta_{f}(R, Z)}{\partial R}\right|_{R=R_{i}}=-\sum_{l=1}^{N} \bar{\theta}_{l}^{*}(Z, t \rightarrow \infty) \frac{\mu_{l}^{2}}{R_{i}} \int_{0}^{R_{i}} R^{\prime} \widetilde{\psi}_{l}\left(R^{\prime}\right) d R^{\prime}$

\section{Results and discussion}

As test case, the flow of air $\left(k_{f}=0.0271 \mathrm{~W} / \mathrm{m}^{\circ} \mathrm{C}, c_{p, f}=1.0049 \mathrm{~kJ} /\right.$ $\mathrm{kgK}, \quad c_{v}, \quad f=0.7178 \mathrm{~kJ} / \mathrm{kgK}, \quad \nu_{f}=1.568 \times 10^{-5} \mathrm{~m}^{2} / \mathrm{s} \quad$ and $\left.\rho_{f}=1.177 \mathrm{~kg} / \mathrm{m}^{3}\right)$ inside an acrylic microchannel $\left(k_{s}=0.2 \mathrm{~W} / \mathrm{m}^{\circ} \mathrm{C}\right)$ is considered, resulting in $K_{s}=k_{s} / k_{f}=7.38$. In the examples presented, it has been adopted $\beta_{v}=1.5, \beta_{t}=2.0$ and $\mathrm{Kn}=0.025$, which are representative values for these parameters $[25,26]$.

Results regarding the solution of the eigenvalue problem given by Eqs. (8a)-(8b,c) are first reported, employing the GITT with integral balance for convergence acceleration in comparison against the traditional solution, without the integral balance scheme. In both cases it has been considered $L_{f i c}=10^{-3}$, resulting in $K_{f i c}=0.04988$. Tables 1(a,b) present the convergence behavior of the first five eigenvalues. It is worth observing that in Table 1(a) the eigenvalues seem to be converged with three to four significant digits for a truncation order as low as $M=50$, for the implementation employing the integral balance approach, whereas in Table 1(b) a very high truncation order of $M=8000$ is needed in order to achieve an agreement of two to three significant digits with Table 1(a), already demonstrating the remarkable convergence acceleration effect obtained with the integral balance approach. Fig. 2 depicts the comparison of the spatial behavior of the first two calculated eigenfunctions, $\psi_{1}(R)$ and $\psi_{2}(R)$, with $M=50$ in both cases, where it can be noticed that the profiles calculated with the integral balance technique present a clear abrupt transition at the interface, $R=R_{i}=0.2$, as result of the solution accurately capturing the fictitious layer which is located from $R=R_{i}$ to $R=R_{i}+L_{\text {fic }}=0.201$ in this case. On the other hand, this multiscale character of the problem poses a significant difficulty for the solution without the convergence acceleration technique, yielding smooth profiles at $R=0.2$. In fact, in order to accurately capture the thin fictitious layer without any kind of convergence acceleration, auxiliary eigenfunctions with much higher frequency would be needed, which would require a prohibitively large truncation order $M$, as demonstrated in Table 1.
Table 2

(a) Convergence behavior for the GITT solution with integral balance $(M=100)$ with (a) $L_{\text {fic }}=10^{-2}$; (b) $L_{f i c}=10^{-3}$.

\begin{tabular}{lllll}
\hline$N$ & \multicolumn{2}{l}{$\theta(R, Z=0.05) \mathrm{Pe}=10$} & \\
\cline { 2 - 5 } & $R=0.1$ & $R=0.2$ (fluid) & $R=0.2$ (solid) & $R=0.6$ \\
\hline (a) & & & & \\
10 & 0.90548 & 0.77921 & 0.74928 & 0.62869 \\
20 & 0.90612 & 0.77957 & 0.74997 & 0.62806 \\
30 & 0.90611 & 0.77950 & 0.74995 & 0.62807 \\
40 & 0.90611 & 0.77949 & 0.74995 & 0.62806 \\
50 & 0.90611 & 0.77949 & 0.74995 & 0.62806 \\
FDM & 0.906 & 0.780 & 0.750 & 0.627 \\
(b) & & & & \\
10 & 0.90627 & 0.77951 & 0.74962 & 0.62812 \\
20 & 0.90681 & 0.77992 & 0.75035 & 0.62749 \\
30 & 0.90681 & 0.77990 & 0.75035 & 0.62750 \\
40 & 0.90681 & 0.77989 & 0.75035 & 0.62750 \\
50 & 0.90681 & 0.77989 & 0.75034 & 0.62750 \\
FDM & 0.906 & 0.780 & 0.750 & 0.627 \\
\hline
\end{tabular}

Table 2(a,b) present the convergence behavior of the calculated temperature field via GITT with integral balance for $L_{f i c}=10^{-2}$ and $L_{f i c}=10^{-3}$, respectively, considering the case with $\mathrm{Pe}=10$ with truncation orders of $N=10$ to $N=50$ in Eq. (9b), employing $M=100$ terms in the solution of the eigenvalue problem with space variable coefficients. Although smaller values of $L_{f i c}$ should in principle lead to better results comparing to the original multi-region problem, it should be remembered that the lower this value, slower becomes the convergence of the eigenvalue problem with multiscale variable coefficients.

In both tables the numerical solution of the original multi-region problem is also presented, obtained via the Finite Difference Method (FDM) [22], employing a second-order accurate scheme in $R$ and firstorder accurate upwind scheme in $Z$. The FDM solution presented was obtained with 1200 nodes in the longitudinal direction and 140 nodes in the radial direction (70 in each region, fluid and solid), yielding

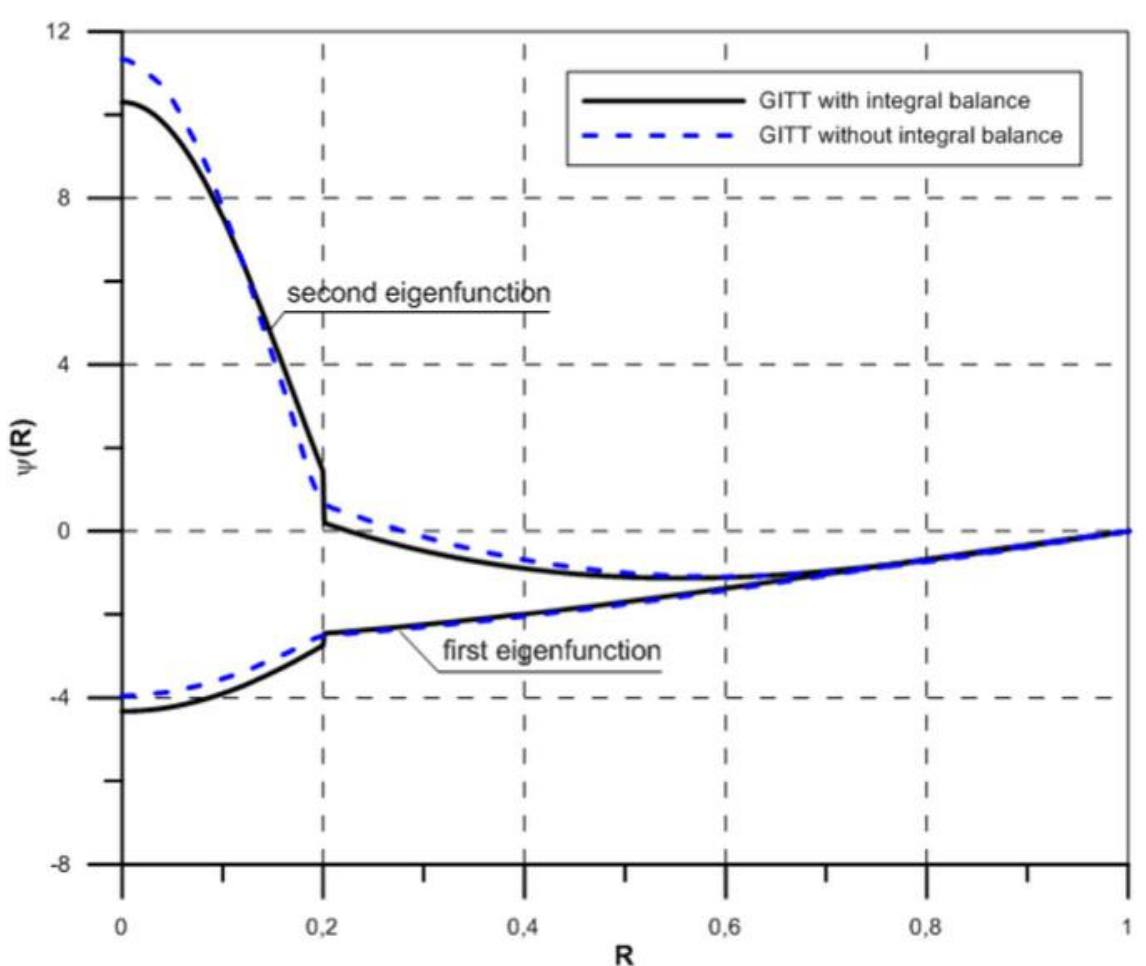

Fig. 2. First two eigenfunctions calculated via GITT with and without convergence enhancement (integral balance approach) employing $L_{f i c}=10^{-3}$ and truncation order of $M=50$ in both solutions. 
(a)

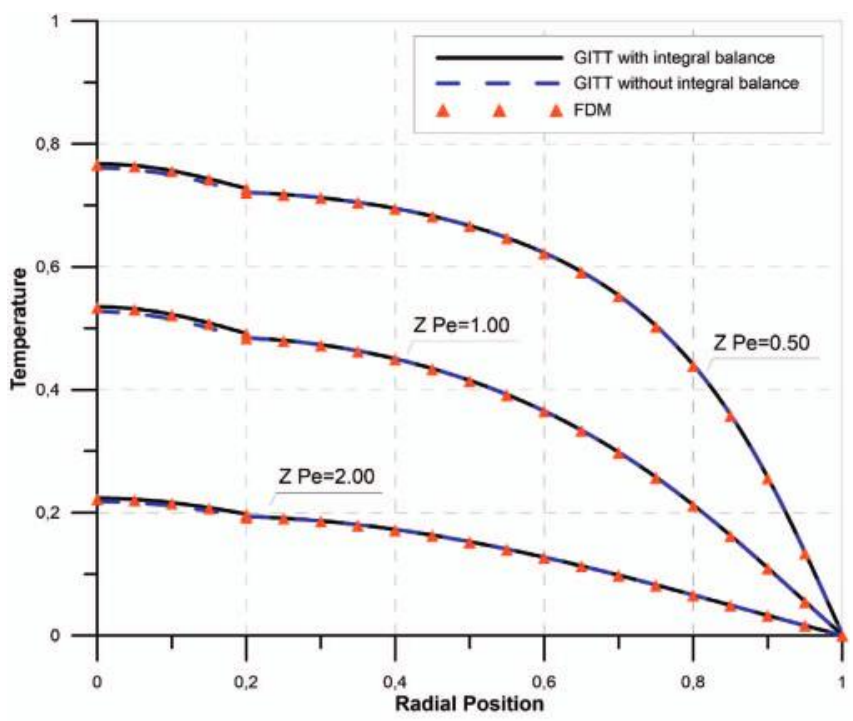

(b)

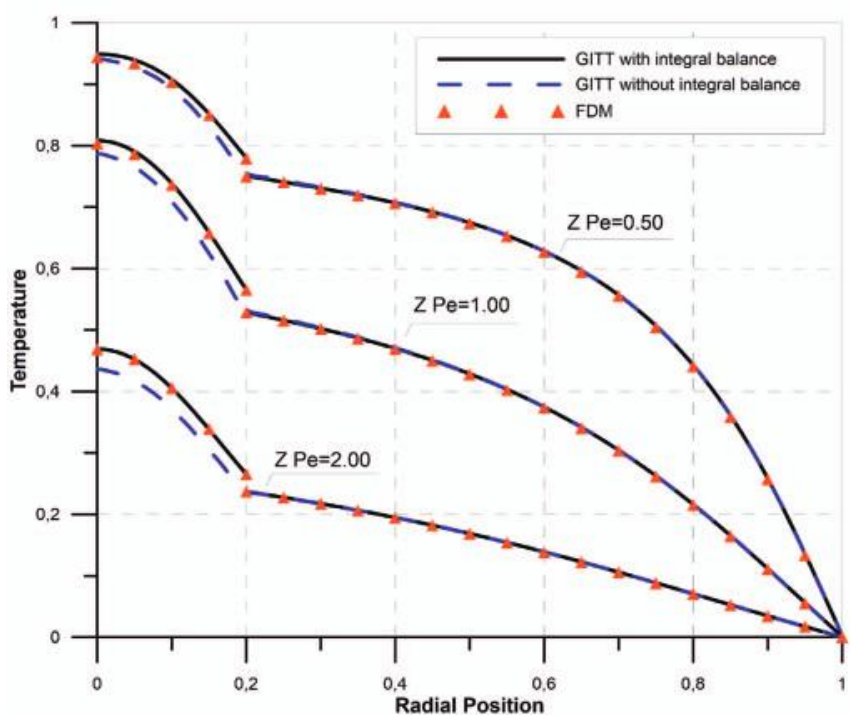

Fig. 3. Comparison of temperature profiles at different longitudinal positions calculated with $L_{f i c}=10^{-3}$ and $M=N=20$ for (a) $\mathrm{Pe}=1$ and (b) $\mathrm{Pe}=10$.

convergence of the three digits shown, confirmed in a grid independence test.

In both cases, $L_{f i c}=10^{-2}$ and $L_{f i c}=10^{-3}$, the GITT solution is converged to five significant digits for the truncation order $N=50$, thus verifying the FDM solution of the original multi-region problem.

Fig. 3(a,b) show temperature profiles calculated with $L_{f i c}=10^{-3}$ and $M=N=20$, at different longitudinal positions for $\mathrm{Pe}=1$ and $\mathrm{Pe}=10$, respectively, confirming the good agreement between the numerical finite differences results and the proposed hybrid solution, while comparing the GITT solutions with and without convergence acceleration of the eigenvalue problem solution. It is interesting to confirm that the most impressive effect of the convergence acceleration technique refers to the interface, allowing for the hybrid solution with the single domain formulation to accurately capture the temperature jump at fairly low truncation orders. Hence, even though the GITT solution without convergence acceleration is still able to yield good results at the solid region, it would require larger truncation orders to accurately treat the interface and the fluid region. It can also be observed that this effect is more pronounced for $\mathrm{Pe}=10$, when a more significant temperature jump occurs.

Some results are now reported in order to investigate the influence of the conjugation effects, Péclet and Knudsen numbers on the asymptotic Nusselt number. In all cases the Nusselt number was calculated employing a fictitious layer with thickness $L_{f i c}=10^{-3}$ and truncation orders $M=N=20$. First, Fig. 4(a,b) and Fig. 5(a,b) depict the calculated asymptotic Nusselt number for different Péclet and Knudsen numbers for (a) $r_{i} / r_{o}=0.5$ and (b) $r_{i} / r_{o}=1$ (no conjugation). In all graphs it is also presented the classical asymptotic Nusselt number (no-slip) for the Graetz problem, if neither conjugation effects nor axial conduction are considered, yielding $\mathrm{Nu}=3.657$. It can be noticed that lower values of the Péclet number lead to slightly higher Nusselt, and for Pe $>20$ the influence of the axial conduction is already very small. On the other hand, increasing the Knudsen number leads to lower Nusselt numbers, in a practically linear behavior for Kn $>0.02$. It can also be readily observed that the conjugation effect (increasing wall thickness, i.e. lower $r_{i} / r_{o}$ ratios) leads to higher Nusselt numbers in this problem, as it can be clearly observed in Figs. 4 and 5, where the curves are shifted up for $r_{i} / r_{o}=0.5$ in comparison with $r_{i} / r_{o}=1$. In all cases, it is worth noting that the Nusselt numbers can be significantly different from the classical asymptotic value, if the effects of slip flow, wall conjugation and fluid axial diffusion are not taken into account.

The influence of the conjugation effect can be observed with more details in Figs. 6(a,b) for a fixed Knudsen number, $\mathrm{Kn}=0.02$, illustrating that the influence of the Péclet number on the Nusselt number is

(a)

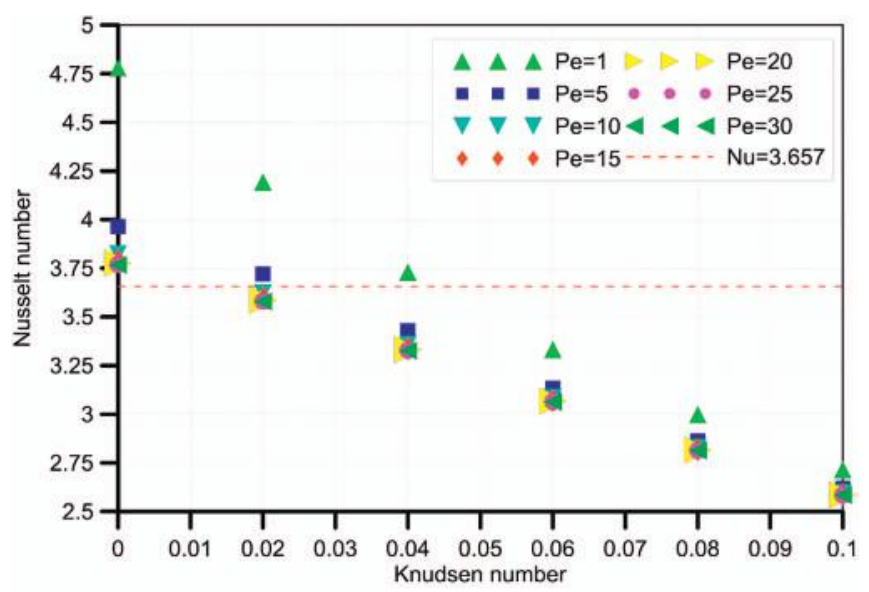

(b)

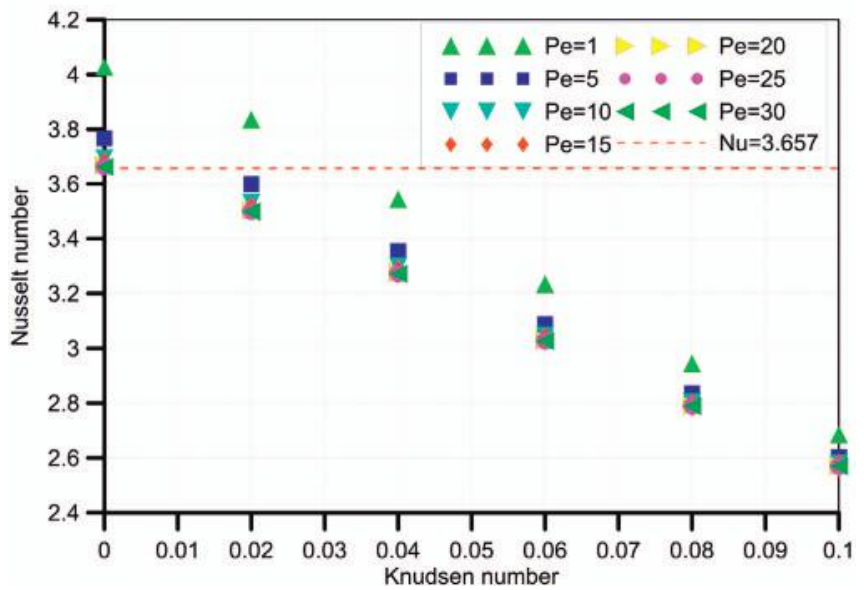

Fig. 4. Asymptotic Nusselt number for different values of Pe and Kn. (a) $r_{i} / r_{o}=0.5$; (b) $r_{i} / r_{o}=1$ (no conjugation). 
(a)

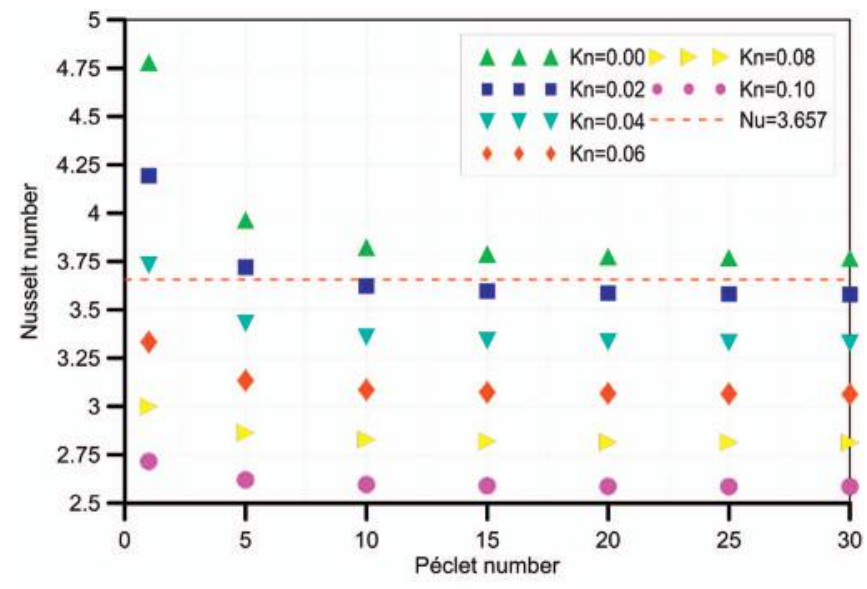

(b)

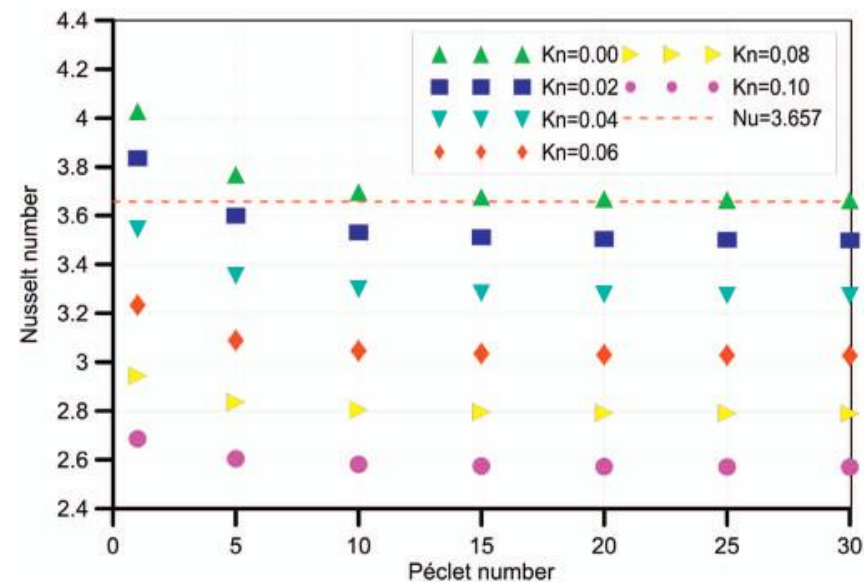

Fig. 5. Asymptotic Nusselt number for different values of Pe and Kn. (a) $r_{i} / r_{o}=0.5$; (b) $r_{i} / r_{o}=1$ (no conjugation)

more pronounced when conjugation effects are important, for lower $r_{i}$ / $r_{o}$ ratios. These results confirm that marked differences are observed if both the axial conduction and the conjugation effects are neglected, being this conclusion especially important on the analysis of microthermal devices, in which low Péclet numbers are typically observed. Hence, neglecting the combined effects can lead to even more significant error in microfluidics simulations, and should be carefully considered whenever the governing parameters are within such ranges.

\section{Conclusions}

Conjugated convection-conduction heat transfer is analyzed in circular microchannels within the slip flow regime and for low Péclet numbers, i.e. considering the axial conduction effect. The Generalized Integral Transform Technique (GITT) is employed in obtaining a hybrid numerical-analytical solution, in combination with a single domain reformulation strategy. The original multi-region problem is rewritten as a single domain with space variable coefficients with abrupt shifts, including a fictitious layer to model the temperature jump at the interface, leading to a multiscale problem. In order to obtain accurate results within sufficiently low truncation orders, an integral balance technique for enhancing the convergence of the eigenfunctions has been employed, recently developed for the solution of eigenvalue problems with multiscale and abrupt transitions. Critical comparisons against the traditional GITT solution (without convergence acceleration of the eigenvalue problem solution) and a dedicated finite difference (a)

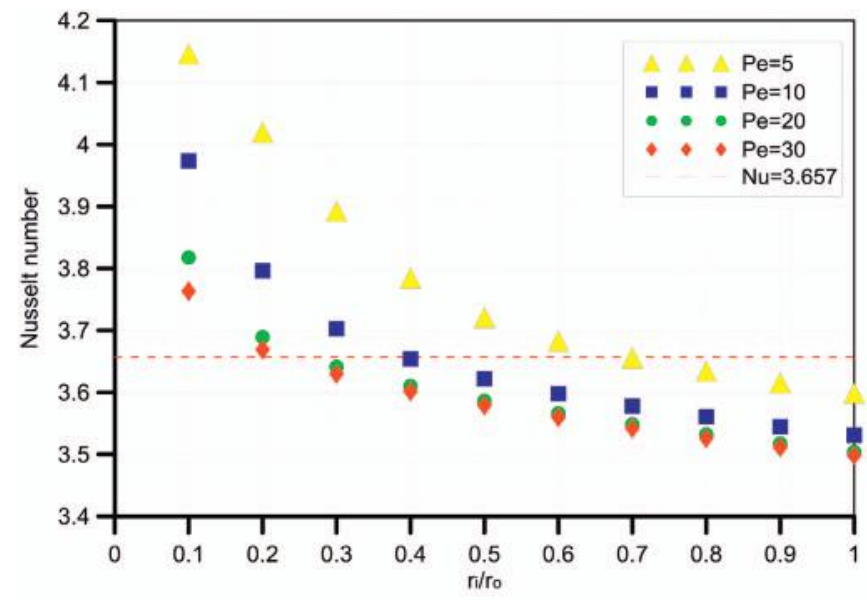

(b)

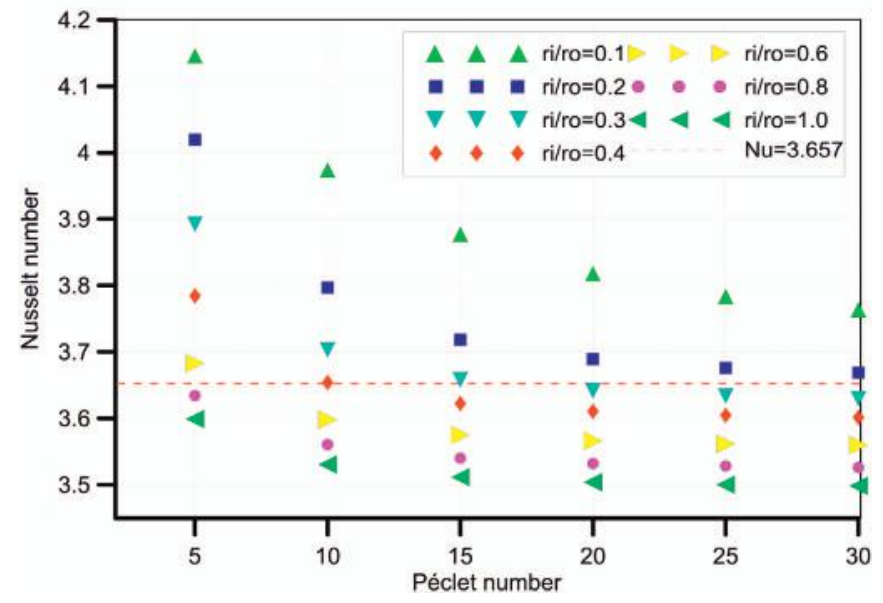

Fig. 6. Asymptotic Nusselt number for different values of Pe and $r_{i} / r_{o}$ (with $\mathrm{Kn}=0.02$ ).

implementation for the original multi-region problem, demonstrate the enhanced convergence behavior and improved accuracy achieved by the proposed methodology. This work also presented a brief analysis of the influence of rarefaction, axial diffusion, and wall conjugation on the estimation of the asymptotic Nusselt number, confirming that marked deviations are observed if their combined effects are neglected, especially for those problems with lower Péclet numbers.

\section{Acknowledgements}

The authors acknowledge the financial support provided by the Brazilian sponsoring agencies CNPq, FAPERJ, and CAPES.

\section{References}

[1] G.L. Morini, Single-phase convective heat transfer in microchannels: a review of experimental results, Int. J. Therm. Sci. 43 (7) (2004) 631-651.

[2] H. Herwig, O. Hausner, Critical view on "new results in micro-fluid mechanics": an example, Int. J. Heat Mass Transf. 46 (5) (2003) 935-937.

[3] R.M. Cotta, D.C. Knupp, C.P. Naveira-Cotta, Analytical Heat and Fluid Flow in Microchannels and Microsystems, Springer, 2016.

[4] T.A. Ameel, X. Wang, R.F. Barron, R.O. Warrington, Laminar forced convection in a circular tube with constant heat flux and slip flow, Microscale Thermophys. Eng. 1 (4) (1997) 303-320.

[5] F.E. Larrodé, C. Housiadas, Y. Drossinos, Slip-flow heat transfer in circular tubes, Int. J. Heat Mass Transf. 43 (15) (2000) 2669-2680.

[6] S. Yu, T.A. Ameel, Slip-flow heat transfer in rectangular microchannels, Int. J. Heat Mass Transf. 44 (22) (2001) 4225-4234.

[7] G. Tunc, Y. Bayazitoglu, Heat transfer in rectangular microchannels, Int. J. Heat Mass Transf. 45 (4) (2002) 765-773. 
[8] M.D. Mikhailov, R.M. Cotta, Mixed symbolic-numerical computation of convective heat transfer with slip flow in microchannels, Int. Commun. Heat Mass Transfer 32 (3) (2005) 341-348.

[9] K. Hooman, A. Ejlali, Effects of viscous heating, fluid property variation, velocity slip, and temperature jump on convection through parallel plate and circular microchannels, Int. Commun. Heat Mass Transfer 37 (2010) 34-38.

[10] D.C. Knupp, C.P. Naveira-Cotta, R.M. Cotta, Theoretical analysis of conjugated heat transfer with a single domain formulation and integral transforms, Int. Commun. Heat Mass Transfer 39 (3) (2012) 355-362.

[11] R.M. Cotta, Integral Transforms in Computational Heat and Fluid Flow, CRC Press, Boca Raton, FL, USA, 1993.

[12] R.M. Cotta, M.D. Mikhailov, Heat Conduction: Lumped Analysis, Integral Transforms, Symbolic Computation, Wiley Interscience, New York, USA, 1997.

[13] D.C. Knupp, C.P. Naveira-Cotta, R.M. Cotta, Conjugated convection-conduction analysis in microchannels with axial diffusion effects and a single domain formulation, J. Heat Transf. 135 (9) (2013) 091401.

[14] D.C. Knupp, C.P. Naveira-Cotta, R.M. Cotta, Theoretical-experimental analysis of conjugated heat transfer in nanocomposite heat spreaders with multiple microchannels, Int. J. Heat Mass Transf. 74 (2014) 306-318.

[15] D.C. Knupp, R.M. Cotta, C.P. Naveira-Cotta, Fluid flow and conjugated heat transfer in arbitrarily shaped channels via single domain formulation and integral transforms, Int. J. Heat Mass Transf. 82 (2015) 479-489.

[16] D.C. Knupp, C.P. Naveira-Cotta, A. Renfer, M.K. Tiwari, R.M. Cotta, D. Poulikakos, Analysis of conjugated heat transfer in micro-heat exchangers via integral transforms and non-intrusive optical techniques, Int. J. Numer. Methods Heat Fluid Flow 25 (6) (2015) 1444-1462.

[17] Y. Kabar, R. Bessaïh, M. Rebay, Conjugate heat transfer with rarefaction in parallel plates microchannel, Superlattice. Microst. 60 (2013) 370-388.
[18] M. Rebay, Y. Kabar, S. Kakaç, M. Rebay, S. Kakaç, R.M. Cotta, Numerical simulation of combined microscale effects on convective heat transfer in single-phase flows, Microscale and Nanoscale Heat Transfer: Analysis, Design, and Application, CRC Press, 2016, pp. 283-308.

[19] D.C. Knupp, C.P. Naveira-Cotta, R.M. Cotta, Conjugated heat transfer in microchannels with slip flow regime via single domain formulation and integral transforms, Proc. of the 7th Int. Symposium on Advances in Computational Heat Transfer, CHT-15, ICHMT, Piscataway, USA, 24-29 May 2015, 2015.

[20] R.M. Cotta, C.P. Naveira-Cotta, D.C. Knupp, Enhanced convergence of eigenfunction expansions in convection-diffusion with multiscale space variable coefficients, Numer. Heat Transfer, Part A 70 (5) (2016) 492-512.

[21] D.C. Knupp, F.S. Mascouto, L.A.S. Abreu, C.P. Naveira-Cotta, R.M. Cotta, Conjugated heat transfer with slip flow in microchannels: single domain integral transforms with enhanced convergence, 16th Brazilian Congress of Thermal Sciences and Engineering. November 07-10, 2016, Vitoria, Brazil, 2016.

[22] M.N. Ozisik, H.R.B. Orlande, M.J. Colaço, R.M. Cotta, Finite Difference Methods in Heat Transfer, 2nd edition, CRC press, Boca Raton, FL, 2017.

[23] S. Wolfram, The Mathematica Book, (2015) (Wolfram Media).

[24] R.M. Cotta, D.C. Knupp, C.P. Naveira-Cotta, L.A. Sphaier, J.N.N. Quaresma, The Unified Integral Transforms (UNIT) algorithm with total and partial transformation, Comput. Therm. Sci. 6 (6) (2014) 507-524.

[25] C.P. Naveira-Cotta, R.M. Cotta, H.R.B. Orlande, Inverse analysis of forced convection in micro-channels with slip flow via integral transforms and Bayesian inference, Int. J. Therm. Sci. 49 (6) (2010) 879-888.

[26] C.P. Naveira-Cotta, Direct-inverse problem analysis in the thermal characterization of microsystems, in: M. Rebay, S. Kakaç, R.M. Cotta (Eds.), Microscale and Nanoscale Heat Transfer: Analysis, Design, and Application, CRC Press, 2016, pp. 369-389. 